\title{
Comparison of Primary and Recurrent Urinary Tract Infections in Children
}

Gül Doğan ${ }^{1}$, Hülya İpek ${ }^{1}$

1. Pediatric Surgery, Hitit University Faculty of Medicine, Çorum, TUR

Corresponding author: Gül Doğan, guldemirdag_982@hotmail.com

\section{Abstract}

\section{Aim}

We aimed to compare the demographic and ultrasound data regarding first-episode urinary tract infections with recurrent infections in children.

\section{Methods}

A total of 509 children aged 0-16 years who were diagnosed to have a urinary tract infection (UTI) as confirmed with positive urinary culture tests were retrospectively investigated. A comparison of baseline parameters, responsible pathogen incidences, and ultrasound findings was made between children who had a single episode of UTI $(n=418,82.1 \%)$ with those having second or more recurrent episodes of urinary tract infection ( $\mathrm{n}=91,17.9 \%)$.

\section{Results}

The mean age of children with a single episode of urinary tract infection was significantly lower than those who had recurrent urinary tract infection $(5.33 \pm 4.38$ vs. $7.01 \pm 4.83$ years, $p=0.003)$. Incidences of Escherichia coli and Enterococcus faecalis was significantly higher in patients with recurrent urinary tract infection than those who had single episode $(n=315,75.4 \%$ vs. $n=80,87.9 \%, p=0.009$ and $n=8,1.9 \%$ vs. $n=9,9.9 \%, p<0.001$, respectively). An abnormal ultrasound was significantly more common in patients with recurrent urinary tract infection than those who had a single episode ( $n=41,54.6 \%$ vs. $n=59,22.7 \%$ ). Increased renal parenchymal echogenicity $(\mathrm{p}=0.002)$, bladder cystitis $(\mathrm{p}=0.01)$ and hydronephrosis $(\mathrm{p}<0.001)$ were significantly more common in patients with recurrent urinary tract infection than those who had a single episode of urinary tract infection.

\section{Conclusion}

Escherichia coli and Enterococcus faecalis were the most common responsible pathogens in recurrent urinary tract infections. Structural changes, such as hydronephrosis and bladder cystitis, are likely to have an important role in the etiology of children with recurrent urinary tract infection.

Received 01/23/2020

Review began 01/30/2020

Review ended 02/12/2020

Published 02/17/2020

\section{(c) Copyright 2020}

Doğan et al. This is an open access article distributed under the terms of the Creative Commons Attribution License CC-BY 4.0., which permits unrestricted use, distribution, and reproduction in any medium, provided the original author and source are credited.
Categories: Pediatrics, Pediatric Surgery, Urology

Keywords: urinary tract infections, recurrent infection, pediatrics

\section{Introduction}

Urinary tract infection (UTI) is a worldwide health problem in the pediatric population, with a prevalence of $5.6-7.0 \%$ in young children presenting acutely ill to primary care [1-3]. It has been estimated that $3-7 \%$ of girls and 1-2\% of boys will be diagnosed with UTI by six years of age, and the disease will recur in $12-30 \%$ of these children. Recurrent UTI was defined as having three or more episodes of symptomatic UTI's within a 12-month-period after the first presentation or two or more episodes within six months. The frequency of recurrent UTI varies depending on the source of data, with the incidence being lower in the primary care departments, whereas higher in the emergency and referral settings $[4,5]$.

Recurrent UTI may cause chronic symptoms such as abdominal pain, nausea, vomiting, and fatigue. However, the most feared complication of recurrent UTI is renal scarring, which leads to the development of hypertension and chronic renal failure [6]. Although, as reported in many studies, recurrent UTI may be predisposed by underlying structural abnormalities, including bowel dysfunction or vesicoureteral reflux disease, it has been recognized that children without reflux or any other predisposing condition were also at risk for development of recurrent UTI $[7,8]$. Recent studies have also demonstrated that antibiotic resistance to common uropathogens is rising due to the increasing rate of extended-spectrum $\beta$-lactamase (ESBL) positivity in Escherichia coli (E. coli), Klebsiella pneumonia (K. pneumonia), and other Enterobacteriaceae species and the resistance rates are even higher in children with recurrent infection.

This report presents an analysis of our institutional data from children presenting with first and recurrent 
episodes of UTI to our outpatient department. We aimed to compare first-episode UTI with recurrent infection regarding demographic and ultrasound characteristics and incidences of responsible uropathogens. We also compare ultrasound findings between first and recurrent episodes to reveal the frequency of structural abnormalities that may be associated with the recurrence of the infection.

\section{Materials And Methods}

The study was approved by the local ethics committee (Date 28th June 2019, Number: 2019 -187). The study was conducted in agreement with the Helsinki declaration for studies involving human participants. This retrospective study was conducted in the outpatient department of a tertiary university hospital, and it was made up of patients presented with a first-episode or recurrent episode of a culture-positive urinary tract infection between May 2012 - March 2019. Since this study was a non-interventional archive study, the need for signed informed consent was waived. Medical archive records of a total of 509 children aged 0-16 years who were diagnosed with UTI were included. The diagnose was confirmed by positive urinary culture tests obtained from midstream urine, catheter or suprapubic aspiration which were retrospectively investigated. Patients with urine culture sampling obtained from urinary bag collection were not included. A comparison regarding baseline parameters, responsible pathogen incidences, and ultrasound findings (available in 334 patients) was made between children who had a single episode of UTI ( $\mathrm{n}=418,82.1 \%)$ with those having second or more recurrent episodes of UTI $(n=91,17.9 \%)$. Patients' files were reviewed for baseline information, clinical data, urine culture tests and urinary ultrasound findings. Urinary tract infection was defined as bacterial growth of 105CFU/ML.

\section{Statistical analysis}

Statistical analyses were performed using SPSS (Statistical Package for the Social Sciences) version 20.0. (IMB Inc., Armonk, USA). Descriptive statistics were reported as mean \pm standard deviation for continuous variables and as frequency and percentage for categorical variables. Categorical variables were compared using the Chi-square test or Fisher exact test where appropriate. Continuous parameters with a normal distribution were compared using independent samples t-test, whereas those not demonstrating normal distribution were compared using the Mann-Whitney test. A p-value of less than 0.05 was considered as statistically significant.

\section{Results}

Baseline characteristics were presented in Table 1 . The mean age of children with a single episode of UTI was significantly lower than those who had recurrent UTI ( $5.33 \pm 4.38$ vs. $7.01 \pm 4.83$ years, p=0.003). Gender distribution was similar between the two groups. ESBL positivity was significantly more common in children with recurrent UTI than in those who had a single episode. ( $n=92,22.0 \%$ vs. $n=34,37.4 \%, p=0.002$ ). 


\section{Cureus}

\begin{tabular}{|c|c|c|c|}
\hline Variable & Primary $(n=418,82.1 \%)$ & Recurrent $(n=91,17.9 \%)$ & p-values \\
\hline Age (years) mean (SD) & $5.33 \pm 4.38$ & $7.01 \pm 4.83$ & 0.003 \\
\hline Females & $287(68.7 \%)$ & $69(75.8 \%)$ & 0.17 \\
\hline ESBL positive & $92(22.0 \%)$ & $34(37.4 \%)$ & 0.002 \\
\hline \multicolumn{4}{|l|}{ Pathogen microorganisms ${ }^{x}$} \\
\hline Escherichia coli & $315(75.4 \%)$ & $80(87.9 \%)$ & 0.009 \\
\hline Klebsiella pneumonia & $52(12.4 \%)$ & $12(13.2 \%)$ & 0.86 \\
\hline Enterococcus faecalis & $8(1.9 \%)$ & $9(9.9 \%)$ & $<0.001$ \\
\hline Proteus mirabilis & $9(2.2 \%)$ & $8(8.8 \%)$ & 0.005 \\
\hline Enterococcus faecium & $8(1.9 \%)$ & $3(3.3 \%)$ & 0.41 \\
\hline Klebsiella oxytoca & $6(1.4 \%)$ & $0(0 \%)$ & 0.59 \\
\hline Morganella morganii & $3(0.7 \%)$ & $3(3.3 \%)$ & 0.07 \\
\hline Pseudomonas aeruginosa & $2(0.5 \%)$ & $3(3.3 \%)$ & 0.04 \\
\hline Staphylococcus aureus & $4(1.0 \%)$ & $0(0 \%)$ & 0.99 \\
\hline Staphylococcus haemolyticus & $3(0.7 \%)$ & $1(1.1 \%)$ & 0.54 \\
\hline Staphylococcus hominis & $2(0.5 \%)$ & $1(1.1 \%)$ & 0.44 \\
\hline Enterobacter aerogenes & $1(0.2 \%)$ & $2(2.2 \%)$ & 0.08 \\
\hline Enterobacter amnigenus & $2(0.5 \%)$ & $0(0 \%)$ & 0.99 \\
\hline Streptococcus agalactiae & $1(0.2 \%)$ & $1(1.1 \%)$ & 0.32 \\
\hline Acinetobacter baumannii & $1(0.2 \%)$ & $0(0 \%)$ & 0.99 \\
\hline
\end{tabular}

\section{TABLE 1: Baseline characteristics of patients $(n=509)$}

ESBL - extended-spectrum $\beta$-lactamase; SD - standard deviation

* Presence of at least one positive culture with the pathogen microorganism

In both groups, Escherichia coli was the most common pathogen, and Klebsiella pneumonia was the second most common pathogen in urine cultures. Incidences of Escherichia coli and Enterococcus faecalis were significantly higher in patients with recurrent UTI than those who had single episode ( $\mathrm{n}=315,75.4 \%$ vs. $\mathrm{n}=80,87.9 \%, \mathrm{p}=0.009$ and $\mathrm{n}=8,1.9 \%$ vs. $\mathrm{n}=9,9.9 \%, \mathrm{p}<0.001$, respectively).

Ultrasound data was available for 259 patients (61.9\%) with a single episode and for 75 patients (82.4\%) with recurrent UTI $(\mathrm{p}<0.001)$. An abnormal ultrasound was significantly more common in patients with recurrent UTI than those who had a single episode ( $n=41,54.6 \%$ vs. $n=59,22.7 \%$ ). Increased renal parenchymal echogenicity ( $\mathrm{p}=0.002)$, bladder cystitis $(\mathrm{p}=0.01)$, and hydronephrosis $(\mathrm{p}<0.001)$ were significantly more common in patients with recurrent UTI than those who had a single episode of UTI (Table 2). 


\section{Cureus}

\begin{tabular}{|c|c|c|c|}
\hline Variable & Primary (n=259, 77.5\%) & Recurrent ( $n=75,22.5 \%)$ & p-value \\
\hline Abnormal & $59(22.7 \%)$ & $41(54.6 \%)$ & $<0.001$ \\
\hline Increased renal parenchymal echogenicity & $2(0.8 \%)$ & $5(6.7 \%)$ & 0.002 \\
\hline Bladder cystitis & $18(6.9 \%)$ & $12(16.0 \%)$ & 0.01 \\
\hline$<3 \mathrm{~mm}$ hyperechoic foci within renal calyces & $10(3.9 \%)$ & $3(4.0 \%)$ & 0.96 \\
\hline Hydronephrosis & $29(11.2 \%)$ & $21(28.0 \%)$ & $<0.001$ \\
\hline Persistent ultrasound findings ( $n=93$ ) & $36(66.7 \%)$ & $19(48.7 \%)$ & 0.08 \\
\hline
\end{tabular}

TABLE 2: Ultrasound findings ( $n=334$, available cases)

\section{Discussion}

The results of our study showed that Escherichia coli was the most common responsible pathogen in both primary and recurrent urinary tract infections whereas Enterococcus faecalis was significantly higher in children with recurrent urinary tract infection than those having their first episode. We think that these findings are in line with those reported in a recent review study, which found a significant association with bowel dysfunction and recurrent urinary tract infection in children [3]. The authors of the study reported that $35 \%$ of children with bladder-bowel dysfunction and $51 \%$ of children with concomitant bladder-bowel dysfunction and vesicoureteral reflux had recurrent urinary tract infection.

Our findings are supported by a large-scale study where a total of 1,045 bacteria identified from urine culture samples obtained from pediatric patients. In that study, Escherichia coli was found in $60.3 \%$ of the samples, and it was followed by Enterococcus faecalis (22.4\%) and Klebsiella spp. (6.5\%) [9]. Similarly, another study reported that Escherichia coli was the most common responsible pathogen (51.5\%), and it was followed by Klebsiella spp. (16.8\%) and Enterococcus spp. (9.9\%) in 202 children aged between two months to 18 years [10].

Our data revealed that about $80 \%$ of patients with recurrent episodes had an ultrasound imaging, and more than half of them had an abnormal ultrasound finding. Hydronephrosis was the most common ultrasound finding, whereas bladder cystitis, which is of pathophysiologic importance, was found significantly more common in patients with the recurrent episode. In line with our findings, Yilmaz et al. [11] reported that recurrent urinary tract infections were significantly more common in patients with renal scarring and structural abnormalities. The authors reported that the rate of recurrent infection was as high as $55 \%$, and the presence of an ultrasound abnormality was $41.2 \%$ in children older than five years.

Our study had several limitations, including the retrospective design, and lack of culture-antibiogram susceptibility data. Although ultrasound data was available in the majority of patients with recurrent episodes, one-third of the patients with single episode did not have ultrasound examinations, and thus an unequivocal comparison of ultrasound data between two groups was not possible.

\section{Conclusions}

We saw that Escherichia coli and Enterococcus faecalis were the most common responsible pathogens in recurrent urinary tract infections. Structural changes, such as hydronephrosis and bladder cystitis, are likely to have an important role in the etiology of children with recurrent urinary tract infections.

\section{Additional Information}

\section{Disclosures}

Human subjects: Consent was obtained by all participants in this study. Hitit University Faculty of Medicine, Çorum, TUR issued approval 2019 -187. Animal subjects: All authors have confirmed that this study did not involve animal subjects or tissue. Conflicts of interest: In compliance with the ICMJE uniform disclosure form, all authors declare the following: Payment/services info: All authors have declared that no financial support was received from any organization for the submitted work. Financial relationships: All authors have declared that they have no financial relationships at present or within the previous three years with any organizations that might have an interest in the submitted work. Other relationships: All authors have declared that there are no other relationships or activities that could appear to have influenced the submitted work.

\section{References}


1. Butler CC, O'brien K, Pickles T, et al.: Childhood urinary tract infection in primary care: a prospective observational study of prevalence, diagnosis, treatment, and recovery. Br J Gen Pract. 2015, 65:217-223. 10.3399/bjgp15X684361

2. O'brien K, Edwards A, Hood K, Butler CC: Prevalence of urinary tract infection in acutely unwell children in general practice: a prospective study with systematic urine sampling. Br J Gen Pract. 2013, 63:156-164. 10.3399/bjgp13X663127

3. Shaikh N, Morone NE, Bost JE, Farrell MH: Prevalence of urinary tract infection in childhood: a metaanalysis. Pediatr Infect Dis. 2008, 27:302-308. 10.1097/INF.0b013e31815e4122

4. Mishra OP, Abhinay A, Prasad R: Urinary infections in children. Indian J Pediatr. 2013, 80:838-843. 10.1007/s12098-013-1118-4

5. Price TK, Dune T, Hilt EE, et al.: The clinical urine culture: enhanced techniques improve detection of clinically relevant microorganisms. J Clin Microbiol. 2016, 54:1216-1222. 10.1128/JCM.00044-16

6. Spencer JD, Schwaderer A, McHugh K, Hains DS: Pediatric urinary tract infections: an analysis of hospitalizations, charges, and costs in the USA. Pediatr Nephrol. 2010, 25:2469-2475. 10.1007/s00467-0101625-8

7. Van Batavia JP, Ahn JJ, Fast AM, Combs AJ, Glassberg KI: Prevalence of urinary tract infection and vesicoureteral reflux in children with lower urinary tract dysfunction. J Urol. 2013, 4:1495-1499. 10.1016/j.juro.2013.02.016

8. Craig JC, Simpson JM, Williams GJ, et al.: Antibiotic prophylaxis and recurrent urinary tract infection in children. N Engl J Med. 2009, 361:1748-1759. 10.1056/NEJMoa0902295

9. Sorlózano-Puerto A, Gómez-Luque JM, Luna-del-Castillo JD, Navarro-Marí JM, Gutiérrez-Fernández J: Etiological and resistance profile of bacteria involved in urinary tract infections in young children . Biomed Res Int. 2017, 2017:4909452. 10.1155/2017/4909452

10. Pouladfar G, Basiratnia M, Anvarinejad M, Abbasi P, Amirmoezi F, Zare S: The antibiotic susceptibility patterns of uropathogens among children with urinary tract infection in Shiraz. Medicine. 2017, 96:e7834. 10.1097/MD.0000000000007834

11. Yılmaz S, Özçakar ZB, Kurt Şükür ED, et al.: Vesicoureteral reflux and renal scarring risk in children after the first febrile urinary tract infection. Nephron. 2016, 132:175-180. 10.1159/000443536 